\title{
Data generation and analysis using spreadsheets
}

\author{
DOUGLAS B. EAMON \\ University of Wisconsin, Whitewater, Wisconsin
}

\begin{abstract}
Modern spreadsheets are powerful and useful tools that can often replace special-purpose programs for generating data and for student analysis of simple statistical problems. The inherent flexibility of spreadsheets makes them especially convenient and extensible. Several templates for generating and analyzing data for $t$ tests and analysis of variance are discussed. Users can specify the $n, \bar{X}$, and $S D$ of two or more groups and then execute macros that generate appropriate data. Overwriting the generated data with "real" values turns the spreadsheet into a data analysis program. Spreadsheets hold promise as valuable instructional supplements for simple designs, but they are less suitable for more complex designs, where special-purpose programs may be more appropriate.
\end{abstract}

The pages of Behavior Research Methods, Instruments, \& Computers contain many references to and descriptions of instructional programs that generate results (quantitative data) resembling data that might be collected under a variety of experimental manipulations (e.g., Eamon, 1980; Fischler, 1980; Keenan \& Keller, 1980; Levy \& Durnin, 1981; Stout, 1974). Virtually all of these programs generate random data from a distribution (usually normal) with a mean and variance determined by some theoretical model. These programs were among the earliest applications of computers in teaching experimental and cognitive psychology, and they have proven to be valuable instructional devices over the years (see Castellan, 1983).

Instructors who wish to use such generated data primarily to demonstrate a statistical procedure or to show the effects of the manipulation of various statistical parameters, however, will encounter difficulties with most of these programs. There are a variety of reasons: the level of measurement may be inappropriate, the models used may be especially complex or contain "hidden" variables (e.g., some of the EXPERSIM models), it may be difficult to create the appropriate design within the model, finding a statistically significant difference may require too many data points for a classroom computational demonstration, the programs may take a long time to run, and so on. Furthermore, although most of these programs allow the instructor to manipulate the means and variances of the data generator so that they will generate usable data, doing so frequently involves a complicated series of stepsoften, the creation of an entirely new model. Even when a new model can be created "on the fly," other problems remain. A particularly important consideration in creating problems for use as classroom examples in teaching statistics is that the instructor may want to simplify compu-

\footnotetext{
This research was supported in part by a Faculty Development Grant from the University of Wisconsin-Whitewater. Requests for reprints should be addressed to D. B. Eamon, Department of Psychology, University of Wisconsin, Whitewater, WI 53190 (e-mail: eamond@uwwvax.uww.edu).
}

tations by using only integers as raw data and may further prefer the means to be integers as well; none of the data generators of which I am aware provide this capability.

Hewett (1985) and, later, Halff (1987) showed how a spreadsheet could be used to demonstrate the effects of manipulating variables in a well-defined model. Their spreadsheet models allowed users to manipulate various parameters (e.g., level of stimulation to neural receptors) and observe the effects of the manipulation (e.g., on transmitter firing rate). Hewett (1985) points out two important advantages of developing spreadsheet applications over special-purpose programs: (1) low cost in terms of development time and effort and (2) the great flexibility of the underlying spreadsheet model.

Both Hewett's and Halff's spreadsheets were, however, static and deterministic: given the same input, the output was always exactly the same-there was no error variance. This characteristic severely limits both the topical content of the spreadsheet model and the usefulness of the simulation as an example for traditional statistical analyses.

An additional and even more serious problem with using spreadsheets to generate example data for statistical analysis is that the spreadsheets are themselves relatively fixed in format. For example, a spreadsheet with three columns (e.g., groups) and 24 rows (e.g., observations) cannot normally be used to generate or analyze data with four columns and 28 rows. Of course, it is possible to copy formulas from row to row and column to column, but doing so is laborious and tricky; inserting or deleting rows from columns that need to be added, for example, requires that the formulas specifying the cells to be summed need to be modified, too.

A number of features of modern spreadsheets can be put to use to solve many of these problems. Blocks identified by their upper left and lower right cell coordinates can be used in formulas rather than specific cell references. Using named blocks [e.g., “@SUM(DATA)”] rather than specific cell references ["@SUM(B22..D48)"] further simplifies some problems. Rows or columns can be added or deleted within blocks without causing errors, 
provided that the upper left and lower right "anchor" cells are not deleted. Blocks can be used in macros to allow a relatively painless way of inserting rows and columns of data, overcoming the straitjacket format of most spreadsheet manipulations. Macro languages provide powerful tools for manipulating the form and function of spreadsheets; simple macros can be easily created using the spreadsheet's "record" mode and can then be stored along with or separately from the spreadsheet itself.

The remainder of this paper discusses several specific implementations of these ideas and provides listings containing templates with examples and macros designed for general use in generating or analyzing data.

\section{Between-Groups $t$ Test}

Generating data that fit a pattern analyzable using a between groups $t$ test entails constructing a spreadsheet template that allows the user to enter the $n$, mean, and standard deviation for two data sets, and then executing a macro that generates the appropriate number of data points. The spreadsheet should also compute and display several intermediate statistics and the $t$.

The layout of such a spreadsheet is shown in Listing 1 . As indicated by the text there, all one needs to do to generate data is to specify the $n \mathrm{~s}$, means, and standard deviations, and then press ALT-G to execute the macro. The macro adjusts the column lengths to the size specified and copies the data generating formulas to the rows in each column.

The elements in the first row contain the formula

$$
\begin{gathered}
\text { @ROUND (@SQRT (-2 * @LN (@RAND)) } \\
\text { * @COS }(2 * @ P I * @ R A N D) \\
* \text { STDEVcSPEC + MEANcSPEC, 0), }
\end{gathered}
$$

where STDEVcSPEC is the standard deviation specified by the user for column $c$ and MEANcSPEC is the mean specified for column $c$. This generates a random integer from a normal distribution with a mean of MEANcSPEC and a standard deviation of STDEVCSPEC.

Rows 2 through $n-1$ contain the formula

$$
\begin{aligned}
& \text { @ROUND (@SQRT }(-2 * \text { * @LN (@RAND)) } \\
& \text { * @COS }(2 * @ P I * @ R A N D) \\
& \text { * STDEVcSPEC + (@COUNT (DATAc) } \\
& \text { * MEANcSPEC - @SUM (DATAc), 0). (2) }
\end{aligned}
$$

Formula 2, based on an anonymous reviewer's suggestion, is the same as Formula 1, except the portion beginning "+ (@COUNT (DATAc)...," which adjusts the mean of the distribution from which the new value will be generated in such a way that the adjusted mean will take into account the deviations produced by the earlier values. "DATAc" in Formula 2 is a named block representing the $n-1$ data values that have been generated up to, but not including, the value to be generated in the current cell. The adjustment is based on a simple algebraic
LISTING 1

Template for Between-Groups $t$ Test Data Generator and Analysis

t-test for Independent Groups (Between Subjects t-test) Data Generat ing/Analys is Temp late

This template generates two columns of $n 1$ and $n 2$ data points using values you spec ify for the means and standard deviations. Enter your ns, means and SOs in the marked block, then press ALT-G to execute the data generat ing macro

To use this template to calculate $t$, first specify the $n$ for each group (means and ranges are irrelevant), then press ALT $-G$ to set up the data table size. Then replace the generated values with your own data. Do not enter spaces to erase data; spaces are treated as 0.

The macro and a table of names (i.e., variables) are given at the end of the spreadsheet. The table of names will not be accurate if you change the number of data points generated. To update it, pos it ion the cursor be low the tit le TABLE Of MAMES. then press /EMH ENTER.

DATA SPECIFICATIONS

N Mean

5 ns, means, \& SDs used to generate data StDe

2 Enter your values, then press ALI-G

GENERATED DATA
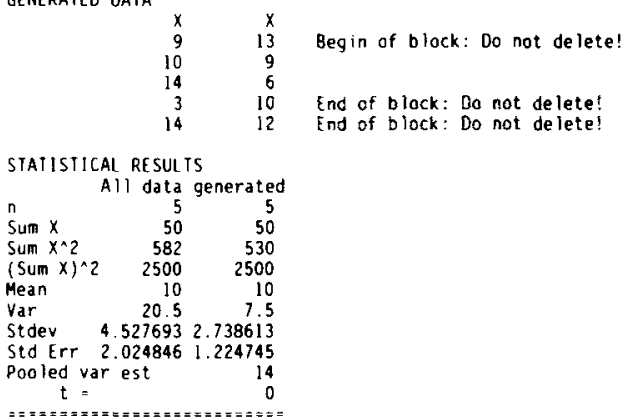

End of block: Do not delete

manipulation of the formula for the mean: if $\bar{X}_{T}$ is the target mean and $n-1$ values have already been generated, the $n$th value will be generated from a distribution having a mean of $n \bar{X}_{T}-\sum_{i=1}^{n-1} X_{i}$. That is, the $n$th value will be generated from a distribution having a mean exactly equal to the value that would, if produced, make the current column mean equal to the target mean.

The formula

$$
\begin{aligned}
& (@ \text { COUNT }(\text { DATAc) }+1) \\
& * \text { MEANcSPEC - @SUM (DATAc) }
\end{aligned}
$$

is placed in the last row of each column to ensure that the column mean will equal the mean specified by the user. This formula does not and should not include the @ROUND() function. It is algebraically transparent, though not necessarily obvious, that if the mean of a distribution is an integer and all but one of the elements of the distribution are integers, then the last element must also be an integer.

One effect of using previous values to adjust the value to be generated next is that, as observed by John Castellan (personal communication, August 1991), since the values are no longer independent, they are not random.

The macro, shown in Listing 2, copies the appropriate formulas into each cell and adjusts the column lengths for unequal $n s$. The spreadsheet automatically generates the data and calculates the statistical results shown below them. 
LISTING 2

Macro (ALT-G) to Copy Formulas to Rows to Generate Data for Between-Groups $t$ test

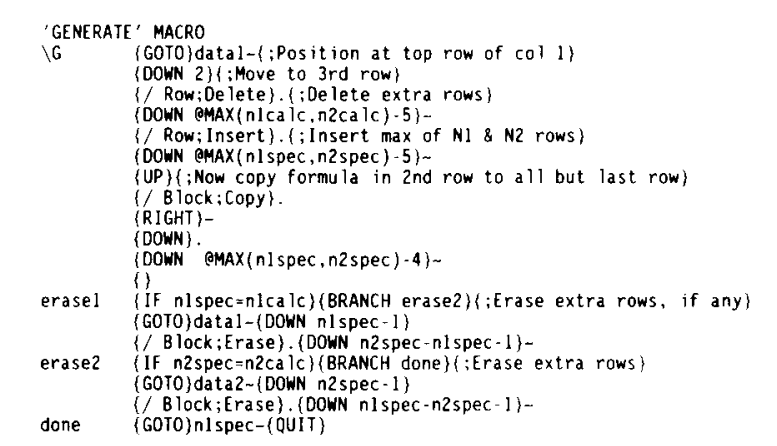

LISTING 3

Within-Groups (Matched Pairs) $t$ Test Template

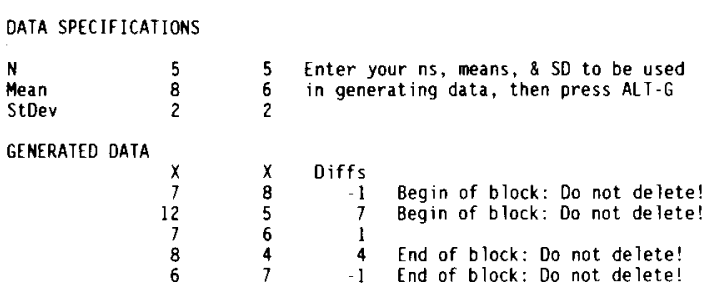

STATISTICAL RESULIS

$\begin{array}{lrrr}n & 5 & 5 & 5 \\ \text { Sum } x & 40 & 30 & 10 \\ \text { Sum } x^{\wedge} 2 & 342 & 190 & 68 \\ \text { (Sum } x)^{\wedge} 2 & 1600 & 900 & 100 \\ \text { Mean } & 8 & 6 & 2 \\ \text { Var } & 5.5 & 2.5 & 12 \\ \text { Stder } & 2.345208 & 1.581139 & 3.464102 \\ \text { Std Err } & 1.048809 & 0.707107 & 1.549193 \\ & & t=1.290994\end{array}$

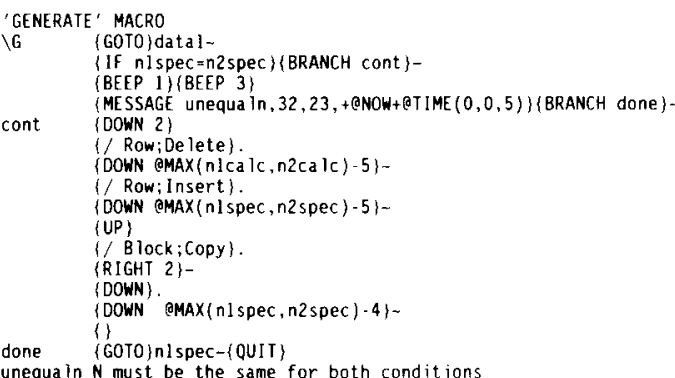

\section{Within-Groups $t$ Test}

The within-groups $t$ test can be done in much the same way as the between-groups $t$ test, and it uses the same general approach in the macro. Listing 3 shows a portion of the template. The major differences are in the inclusion of a column of differences and the statistical evaluation of that column to produce the $t$ value. As also shown in Listing 3, the macro that copies the rows also copies this column. Rather than delete rows to match the specifications for $N_{1}$ and $N_{2}$, the macro ensures that the columns are of equal lengths at the outset and delivers a message if they are not.
One-Way Between-Groups Analysis of Variance

A more complex scheme is required for analysis of variance (ANOVA). In order to make the spreadsheet template most general and flexible, it must be possible to specify the number of groups (columns) before specifying the $n \mathrm{~s}, \bar{X} \mathrm{~s}$, and $S D \mathrm{~s}$ for each column. Thus, two macros are needed: one to allow the user to specify the number of columns and a second to specify the column parameters. Listing 4 shows a template in which the user has specified the number of columns and rows but has not executed the macro that generates the columns. Listing 5 shows the same template after the user has executed a macro that creates the columns. Listing 6 shows the template after the user has entered the column parameters and has executed the data generating macro.

The macro that creates the columns (ALT-K) is shown in Listing 7, and the macro that copies the formulas to the rows (ALT-G) is shown in Listing 8.

\section{Repeated Measures and Complex Analysis of Variance}

Procedures for generating data for repeated measures ANOVA are similar to those used for between-groups ANOVA, and they will not be discussed here. Interested readers should contact the author to receive templates and macros for these designs.

Templates and macros for other more complicated ANOVA procedures, including two- and three-factor designs, can also be created. At some point, however, these more complex designs begin to subvert the rationale for using the spreadsheet; they become so complex that the simplicity and intuitive character of the spreadsheet ap-

\section{LISTING 4}

\section{Between-Groups ANOVA Before Columns Have Been Created}

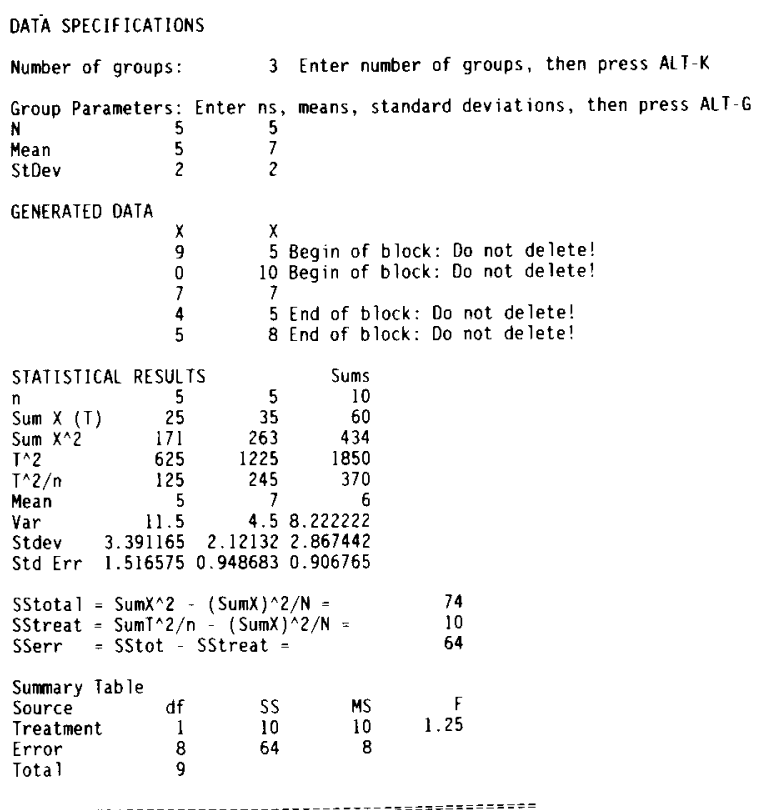


LISTING 5

Between-Groups ANOVA After User Has Generated Columns and Specified Group Parameters

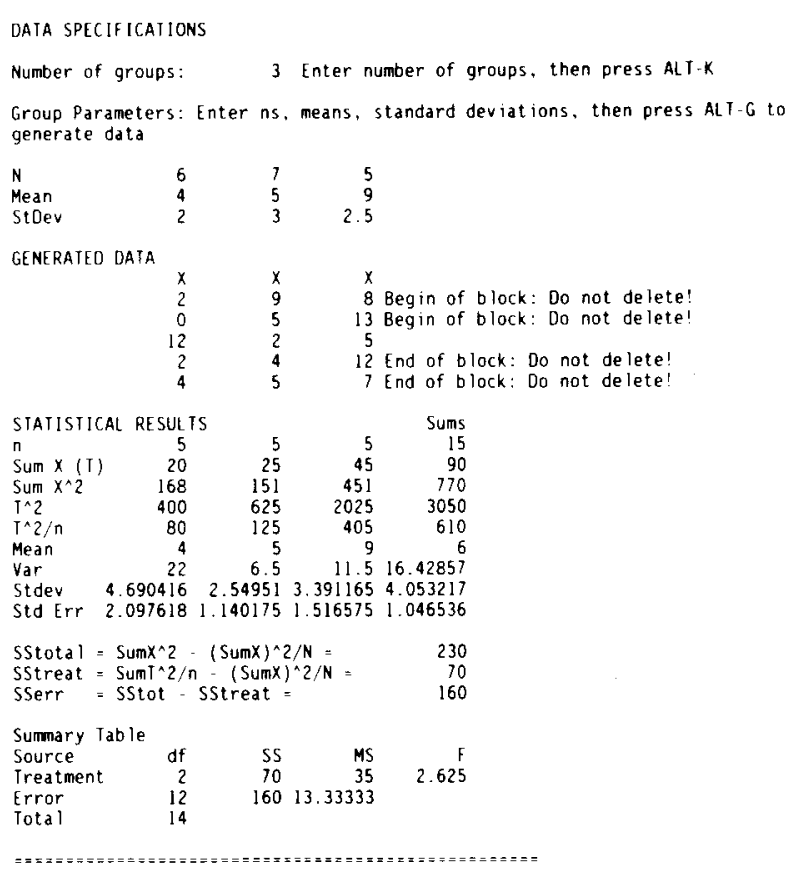

proach is lost. It is possible to create them using the spreadsheet, but it is rare that one would want to generate data for, say, a three-factor design. Specialized programs, such as Bradley et al.'s (1992) DATASIM, provide a better alternative for data generation, and more powerful statistical analysis programs would be more appropriate and convenient for data analysis in these cases.

\section{Other Considerations}

Analysis of "real" data. Spreadsheet templates can also be used for analysis of "real" data or data collected from other sources. To do so, it is only necessary to specify the $n$ s for each column, press ALT-G to adjust the column lengths, and then enter the new data values over the top of the ones generated by the program. The user may defer all computations until later by resetting the spreadsheet's recalculation frequency to manual. With a reasonably fast computer and a small data set, however, students find it interesting and instructive to see these values recalculated with each data point entered.

"SOLVE FOR" function. More powerful spreadsheets include a "SOLVE FOR" command that allows the user to specify a desired result of a formula and indicate a cell referenced by the formula; the spreadsheet will attempt to adjust the value of the referenced cell so that the result is as specified. This suggests that to find data sets with particular characteristics one might expect to be able to specify a set of $N-1$ "fixed" data points and then ask the spreadsheet to select an $N$ th value that would yield, say, a specified $F$ value. Although this was partly suc- cessful for intermediate results, such as the sums of squares and even variances, it was generally unworkable for "higher level" results unless the variable cell was already very close to the target value.

Noninteger values. Because integer values are particularly desirable for instructional examples, the datagenerating templates discussed above assumed that the $@$ ROUND() function sets 0 decimal places. However, nothing about any of the computational techniques or the macros restricts the number of decimal places to 0 ; to change the number of decimal places, all that needs to be done is to change the 0 decimal places in the @ROUND() function in the first four rows of the template. Those who wish to make such changes should be reminded that the first row of each column differs from the second through $n-1$ st, which is different from the $n$th row, as noted above.

Including other or additional results. It is possible to include more or fewer intermediate statistical results in the templates. If removing intermediate values invalidates later results that depend on the earlier ones, it is usually possible simply to modify the formula in the later result so that they include the earlier ones. Any changes in the number of intermediate results will cause problems unless the macros' commands that copy the columns are modified.

User-defined statistics. Although this paper does not provide instructions on writing cell formulas or macros for spreadsheets, the examples provided are sufficiently detailed and heavily commented, and they may serve as

\section{LISTING 6}

Between-Groups ANOVA After User Has Generated Data

$$
\begin{aligned}
& \text { DATA SPECIFICATIONS } \\
& \text { Number of groups: } \quad 3 \text { Enter number of groups, then press ALI-K } \\
& \text { Group Parameters: Enter ns, means, standard deviations, then press ALT.G to }
\end{aligned}
$$

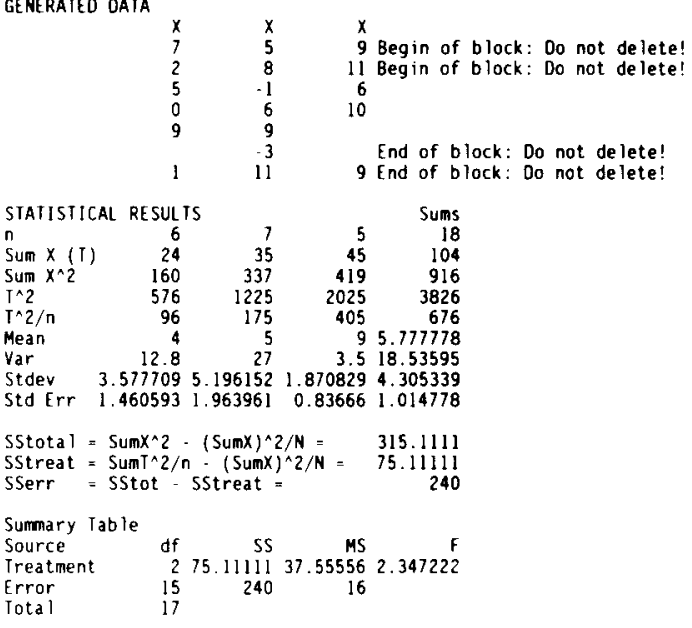


LISTING 7

Macro to Create Columns for Between-Groups ANOVA

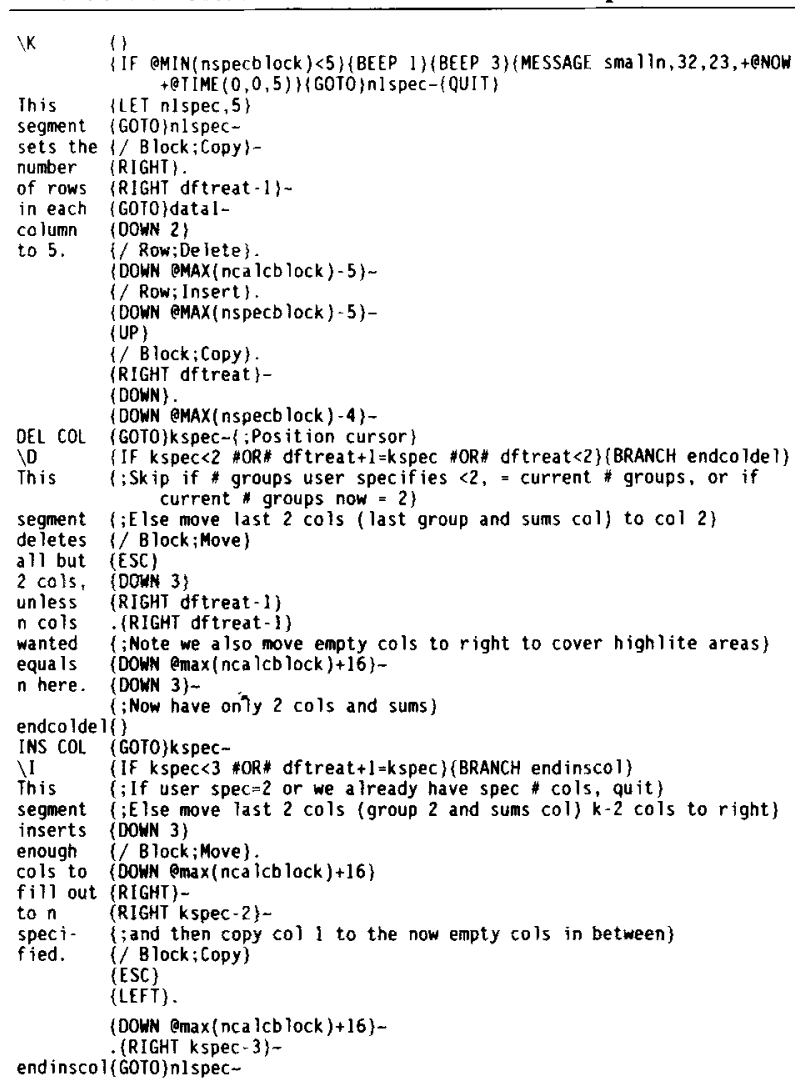

LISTING 8

Macro to Generate Data for Between-Groups ANOVA

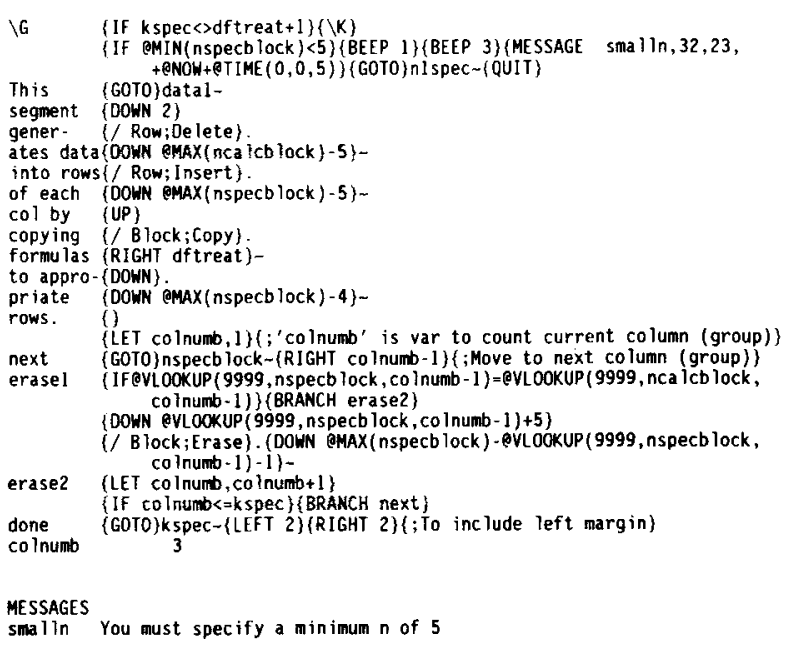

examples of how a spreadsheet template might be constructed for many statistical procedures. The most difficult aspect of writing the macros is providing for maximum flexibility; the approach chosen here is to fix the formulas in one column and a few rows and then copy these formulas to additional columns and rows as needed. An alternative, perhaps safer, procedure would be to have the macro write in the formula for each important cell before copying. This would protect the formula from alteration by the user, as well as provide greater flexibility than that provided by the approach here. Such macros would, however, be considerably more complex and more difficult to debug than those used here.

Macro languages as programming languages. Although the spreadsheet's statistical work is done in the cells by formulas there, it is the macros that provide the power to make the spreadsheet templates flexible and usable. While spreadsheet macro languages tend to be obscure in syntax and clumsy in implementation, it is relatively simple to create them. Most spreadsheets can record user keystrokes and save them as a macro. Once debugged, they can be stored in separate macro libraries and called up to any open spreadsheet. Unlike the programming languages with which most psychologists have had experience, however, spreadsheet macro languages tend to require a rigid syntax and are quite unforgiving in comparison. However, the wide variety of applications for which spreadsheets may be used and the commonality of these languages across disciplines (it has been said that the Lotus 1-2-3 macro language is the most widely known programming language in use today) make such languages a valuable asset for instructors and students. Students who have taken a general computer course may already be familiar with a spreadsheet macro language and may be able to construct their own templates for statistical analysis.

\section{Summary and Availability}

Spreadsheets are a widely available tool that can be used to generate and analyze data. The data-generating possibilities have wide-ranging potential. Data analysis using spreadsheets can be an interesting and instructive exercise with an already familiar tool but cannot substitute for a good statistical package. Macro languages provide great flexibility, although they are often cumbersome and finicky.

The spreadsheet templates discussed here may be obtained from the author without cost. Please include a formatted (MS-DOS only) disk.

\section{REFERENCES}

Bradley, D., Hemstreet, R. L., \& Ziegenhagen, S. T. (1992). A simulation laboratory for statistics. Behavior Research Methods, Instruments, \& Computers, 24, 190-204.

Castellan, N. J. (1983). Strategies for instructional computing. Behavior Research Methods \& Instrumentation, 15, 270-279.

EAMON, D. (1980). Labsim: A data-driven simulation program for instruction in research design and statistics. Behavior Research Methods \& Instrumentation, 12, 160-164. 
Fischler, I. (1980). An on-line laboratory in cognition and perception. Behavior Research Methods \& Instrumentation, 12, 116-119.

HALFF, L. (1987). A spreadsheet simulation of the early stages of visual information processing. Behavior Research Methods, Instruments, \& Computers, 19, 117-122.

HEwETT, T. (1985). Teaching students to model neural circuits and neural networks using an electronic spreadsheet. Behavior Research Methods, Instruments, \& Computers, 17, 339-344.
Keenan, J., Keller, R. (1980). Teaching cognitive processes: Software for laboratory instruction in memory and cognition. Behavior Research Methods \& Instrumentation, 12, 103-110.

LevY, M., Durnin, M. (1981). Modeling on microcomputers. Behavior Research Methods \& Instrumentation, 13, 201-203.

Stout, R. (1974). Modelling and the Michigan experimental simulation supervisor. Behavior Research Methods \& Instrumentation, 6 , $12 !-123$ 